\title{
Control of reflexive and voluntary saccades in the gap effect
}

\author{
GREGORY L. CRAIG \\ Carleton University, Ottawa, Ontario, Canada \\ and \\ LEW B. STELMACH and W. JAMES TAM \\ Communications Research Centre, Ottawa, Ontario, Canada
}

\begin{abstract}
In two experiments, we examined whether voluntary and reflexive saccades shared a common fixation disengagement mechanism. Participants were required to perform a variety of tasks, each requiring a different level of information processing of the display prior to execution of the saccade. In Experiment 1, participants executed either a prosaccade or an antisaccade upon detecting a stimulus array. In Experiment 2, participants executed a prosaccade to a stimulus array only if the array contained a target item. The target could be a line (easy search) or a digit (difficult search). The critical manipulation in both experiments was the relative timing between the removal of the fixation stimulus and the onset of the stimulus array. In both experiments, it was found that saccadic latencies were shortest when the fixation stimulus was removed before the onset of the stimulus array-a gap effect. It was concluded that reflexive and voluntary saccades share a common fixation disengagement mechanism that is largely independent of higher level cognitive processes.
\end{abstract}

During the average workday, a person typically makes between 100,000 and 200,000 saccadic eye movements (Irwin \& Carlson-Radvansky, 1996). These can be voluntary, planned by the viewer to examine stimuli of interest, or reflexive, executed in response to novel or abrupt stimuli. Research on saccadic control has focused primarily on reflexive saccades (Fischer, 1987; Fischer \& Ramsperger, 1986; Fischer \& Weber, 1993; Kingstone \& Klein, 1993a, 1993b; Klein, Taylor, \& Kingstone, 1995; Reuter-Lorenz, Hughes, \& Fendrich, 1991; ReuterLorenz, Oonk, Barnes, \& Hughes, 1995; Saslow, 1967; Tam \& Ono, 1994; Tam \& Stelmach, 1993; Weban-Smith \& Findlay, 1991; Weber, Aiple, Fischer, \& Latanov, 1992; Weber, Biscaldi, \& Fischer, 1995). More recently, there have been a number of studies on control of voluntary saccades (Abrams, Oonk, \& Pratt, 1998; Fischer \& Weber, 1992; Forbes \& Klein, 1996; Reuter-Lorenz et al., 1991; Reuter-Lorenz et al., 1995). The goal of the present research is to continue this investigation of saccadic control and to explore differences and similarities in the control of reflexive and voluntary saccades.

A ubiquitous finding with reflexive saccades has been that their latency is reduced by removing a fixation stim-

The authors thank the Natural Sciences and Engineering Research Council of Canada (NSERC) and the Communications Research Center (CRC). Correspondence concerning this article should be addressed to G. L. Craig, Institute for Aerospace Research, Flight Research Laboratory, National Research Council of Canada, Bldg. U-61, Uplands, Ottawa, ON K1A 0R6, Canada (e-mail: greg.craig@nrc.ca).

-Accepted by previous editor, Myron L. Braunstein ulus (gap conditions), as compared with cases in which the fixations stimulus remains visible (overlap condition; Saslow, 1967). The reduction in latency caused by removing the fixation stimulus is known as the gap effect (Kingstone \& Klein, 1993b; Reuter-Lorenz et al., 1991; L. E. Ross \& S. M. Ross, 1980; Tam \& Ono, 1994; Tam \& Stelmach, 1993).

General agreement exists in the literature that the gap effect can be attributed to fixation disengagement (Fischer \& Weber, 1993; Kingstone \& Klein, 1993a, 1993b; Klein et al., 1995; Reuter-Lorenz et al., 1991; Reuter-Lorenz et al., 1995; L. E. Ross \& S. M. Ross, 1980; Saslow, 1967; Tam \& Ono, 1994; Tam \& Stelmach, 1993; Weber et al., 1992; Weber et al., 1995). Reflexive saccades can be executed more rapidly in gap conditions because the offset of the fixation stimulus leads to deactivation of the system responsible for maintaining fixation. Without competition from the fixation system, the saccade system can respond faster to a peripheral stimulus (Forbes \& Klein, 1996; Munoz \& Wurtz, 1992, 1993a, 1993b; Tam \& Ono, 1994).

Fischer (1987) has proposed a three-loop model to explain the gap effect. Reflexive saccades occur via the first loop involving the retina, visual area 1 (V1), the brainstem eye movement centers, and the superior colliculus. Voluntary saccades involve additional processes in the frontal eye fields (first loop) or in the parietal lobe (third loop). This model predicts a gap effect for both reflexive and voluntary saccades, because it assumes a common control structure in which the execution of all saccades depends on the disengagement of the fixation mechanism located in the superior colliculus. If the fixation system in the superior colliculus is disengaged, both reflexive 
and voluntary saccades would be executed sooner than if the fixation system were engaged. The prediction of the three-loop model for reflexive saccades has been well established. Studies of voluntary saccades with the antisaccade task, on the other hand, have yielded mixed results. In the antisaccade task, participants were required to make a saccade to the side of fixation opposite the target (Hallett \& Adams, 1980). Some researchers have found a gap effect (Fischer \& Weber, 1993; Forbes \& Klein, 1996), whereas others have not (Reuter-Lorenz et al., 1991; Reuter-Lorenz et al., 1995).

The unreliability of the gap effect with the antisaccade task may be due to the conflicting demands of the task; reflexive saccades to the visual target must be suppressed, and a saccade must be programmed in the opposite direction. This may increase the variability of the distribution of saccadic latencies. Also, as has been suggested by Forbes and Klein (1996), successful performance in the antisaccade task may require tonic inhibition of the superior colliculus, which overrides the disengaging effect of fixation offset, making the gap effect smaller and more difficult to detect.

Given the problems associated with the antisaccade task, a new task was developed by Forbes and Klein (1996) that used verbal cues to direct saccades to the left and to the right of fixation. Gap effects were found in the verbal cuing task, leading Forbes and Klein to propose a model of fixation control similar to that of Fischer (1987), incorporating the neurophysiological work of Munoz and Wurtz (1993a, 1993b). Both the model of Forbes and Klein and the three-loop model of Fischer are elaborations of a general view of oculomotor control that has been referred to by such phrases as "break or release of fixation" (Lynch, Mountcastle, Talbot, \& Yin, 1977, p. 376) or "free the gaze from the stimulus" (Hallett \& Adams, 1980, p. 331).

The current research continues the investigation of the control of voluntary saccades, exploring the prediction that the gap effect should occur regardless of the extent of planning or higher level processing that might be involved in preparing a saccade. To test this prediction, participants were required to perform a variety of saccadic tasks, each requiring a different level of information processing of the display prior to execution of the saccade. If a gap effect were found across a range of tasks, this would provide strong support for a common control structure and for the general role of fixation disengagement in executing saccades.

Two experiments are reported. The first experiment studied saccadic latencies using (reflexive) prosaccade and (voluntary) antisaccade conditions, providing a common reference point with previous work (Fischer \& Weber, 1992; Forbes \& Klein, 1996; Reuter-Lorenz et al., 1991; Reuter-Lorenz et al., 1995). In the prosaccade condition, participants were required to execute a saccade as quickly as possible to a stimulus array that appeared to the right or the left of fixation (see Figure 1). In the antisaccade condition, the stimulus array appeared on one side of a display, and participants were required to execute a saccade to the opposite side.
The second experiment studied saccadic latencies in a visual search task. Participants were required to search an array for a target item before executing a saccade. Compared with the antisaccade task, the search task is more natural, and it complements the verbal cuing task of Forbes and Klein (1996). In one variant of the search task, a single array was presented on one side of fixation. In another variant, two arrays were presented, one on each side of fixation. As compared with the unilateral presentation, bilateral presentation required participants both to detect the target and to identify the side containing the target. The participants were required to execute a saccade only if a target was presented. Difficulty of search was manipulated by varying the discriminability of the target and distractor items (lines:easy and digits:hard). According to the models of Fischer (1987) and of Forbes and Klein, the mechanisms responsible for fixation disengagement, located in the rostral pole of the superior colliculus, should be largely unaffected by higher level processing requirements. As such, the magnitude of the gap effect should be roughly constant at all combinations of search difficulty and number of arrays.

\section{GENERAL METHOD}

\section{Participants}

There were 8 participants. Each participant served in both experiments. Seven participants were naive observers, whereas I participant had some familiarity with the gap paradigm.

\section{Materials and Displays}

All the displays were presented on a Tektronix 608 oscilloscope equipped with a P15 phosphor and controlled by an Interactive Electronic Systems controller (Finley, 1985). The P15 phosphor has an extremely short persistence and decays to $10 \%$ of its original value in $2 \mu \mathrm{sec}$. The background luminance of the display was $50 \mathrm{~cd} / \mathrm{m}^{2}$, and the luminance of the stimuli was $400 \mathrm{~cd} / \mathrm{m}^{2}$.

The stimulus array consisted of three items presented within an area $1.5^{\circ}$ wide $\times 1.7^{\circ}$ high. The center of the array was $2.5^{\circ}$ to the left or the right of a central fixation stimulus. The individual items in the stimulus display consisted of a 2 and two $5 \mathrm{~s}$, or of a vertical line and two horizontal lines (see Figure 1). Numeric stimuli subtended a visual angle of $0.4^{\circ} \times 0.6^{\circ}$. Line stimuli were $0.6^{\circ}$ long and $0.08^{\circ}$ thick.

The experiments employed unilateral and bilateral presentations. In the unilateral presentations, the stimulus array was presented on one side of fixation. In the bilateral presentations, two arrays were presented, one on each side of fixation.

A headrest was used to maintain a steady head position. Eye position was sampled at a rate of $60 \mathrm{~Hz}$ with an ISCAN RK-426 pupiltracking system. The $60-\mathrm{Hz}$ sampling rate ensured that detection of saccades was accurate to within $16.7 \mathrm{msec}$. The spatial resolution of the eye tracker was better than $0.25^{\circ}$. All the equipment was controlled by a microcomputer.

\section{Procedure}

Each participant viewed the display from a distance of $46 \mathrm{~cm}$. The participants initiated each trial by pressing a button, at which time the central fixation dot was changed to a fixation cross, ensuring that the participant's fixation mechanisms were fully engaged on the fixation stimulus. Four hundred msec after pressing the button, a 100-msec warning tone was presented (see Figure 2). The participants were informed that the warning tone always pre- 


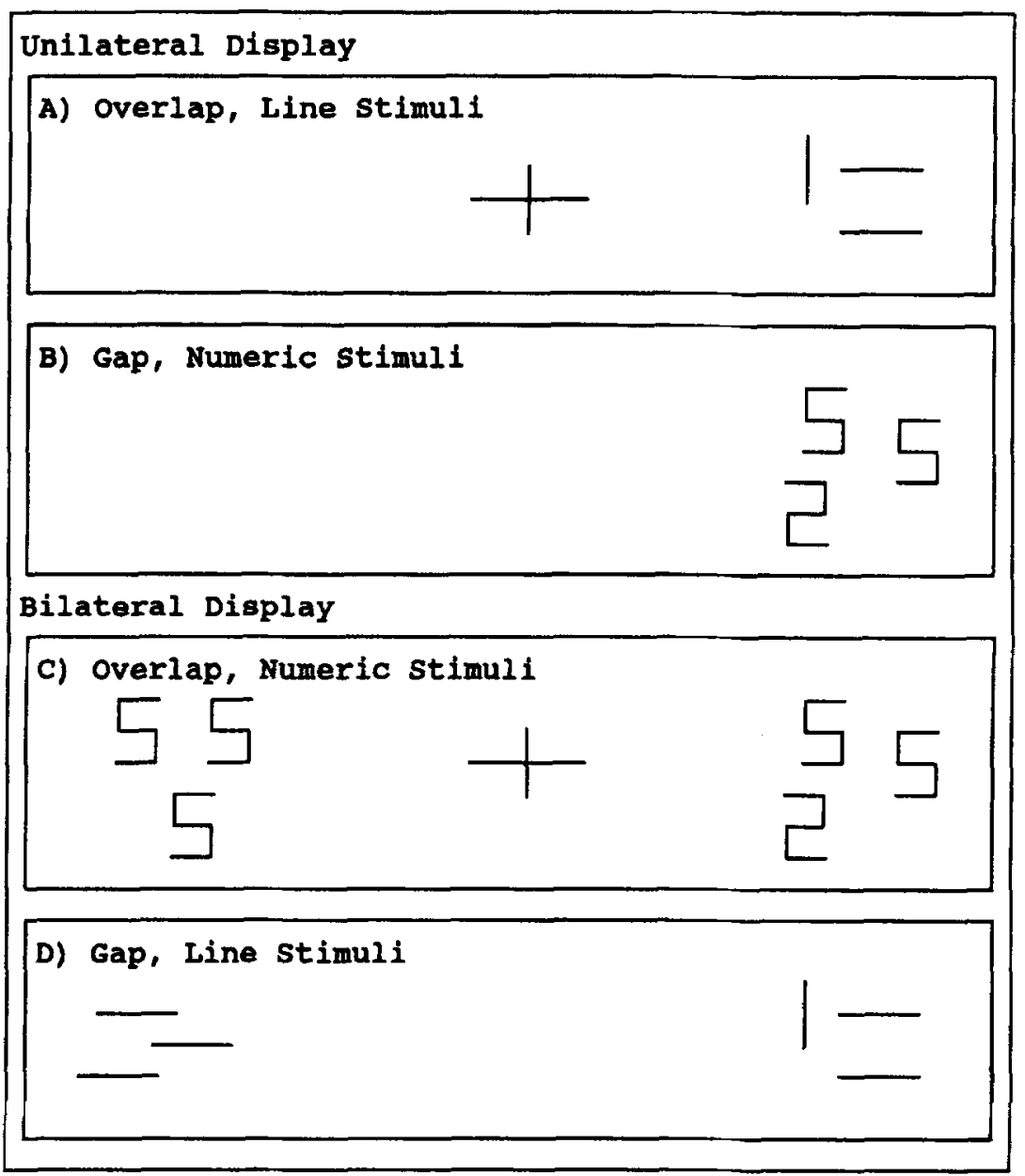

Figure 1. Sample displays used in the experiments. In Experiment 1 , all the displays were unilateral (panels $A$ and B), whereas in Experiment 2, displays were either unilateral or bilateral (panels $C$ and D). Examples of overlap trials are presented in panels $A$ and $C$, whereas examples of gap trials are presented in panels $B$ and $D$.

ceded the onset of the stimulus array by $500 \mathrm{msec}$. The stimulus array(s) appeared $1,000 \mathrm{msec}$ after initiation of a trial. The fixation cross disappeared at variable interstimulus intervals (ISIs) after initiation of the trial. Eight levels of ISI were used $(-500,-100,0,100$, $150,200,300$, and $500 \mathrm{msec}$ ), referring to the interval between the offset of the fixation cross and the onset of the stimulus array. Negative ISIs indicate trials in which the stimulus array and the fixation cross overlapped in time (i.e., onset of the stimulus array occurred while the fixation cross was still present). Positive ISIs indicate trials in which a temporal gap existed between the offset of the fixation cross and the onset of the stimulus array (i.e., onset of the stimulus followed the offset of fixation). At ISI $=0 \mathrm{msec}$, offset of fixation and onset of the stimulus array coincided.

Catch trials, described in detail below, were included to discourage anticipatory saccades to the stimulus array. The number of catch trials per session ranged from 9 to 16 trials (in addition to the 32 target-present trials). The number of catch trials per session was not fixed, in order to reduce their predictability.

Trials were rejected (1) if saccades were too fast (below $85 \mathrm{msec}$, based on Fischer \& Ramsperger, 1984, 1986) or too slow (above $600 \mathrm{msec}$ ); (2) if saccades were too small (less than $1.75^{\circ}$; the minimum edge-to-edge distance from the fixation stimulus to the stimulus array); (3) if no saccade was made when a stimulus array was presented; (4) if a saccade was made in the wrong direction; and (5) if a saccade was made on a catch trial. Rejected trials, not including catch trials, were repeated within the same session. An entire session was repeated if the percentage of rejected trials exceeded $20 \%$.

Each session consisted of 32 target-present trials, 4 trials for each of the eight ISIs. Each participant served in six sessions.

\section{Tasks}

Detection Tasks in Experiment 1. The experiment had three factors: saccade direction (prosaccade vs. antisaccade), ISI (eight levels), and stimulus type (numeric vs. line). Saccade direction and stimulus type were blocked within a session. The dependent measure was saccadic latency.

A stimulus array was presented on the left or the right side of fixation equally often. In prosaccade blocks, the participants were instructed to make a saccade to the center of the array as quickly as possible. In antisaccade blocks, the participants were required to execute a saccade to the side of the display opposite to that containing the stimulus array (Hallett $\&$ Adams, 1980). On catch trials, the stimulus array was not presented.

Search Tasks in Experiment 2. The experiment had three factors: display type (unilateral vs. bilateral), ISI (eight levels), and search 


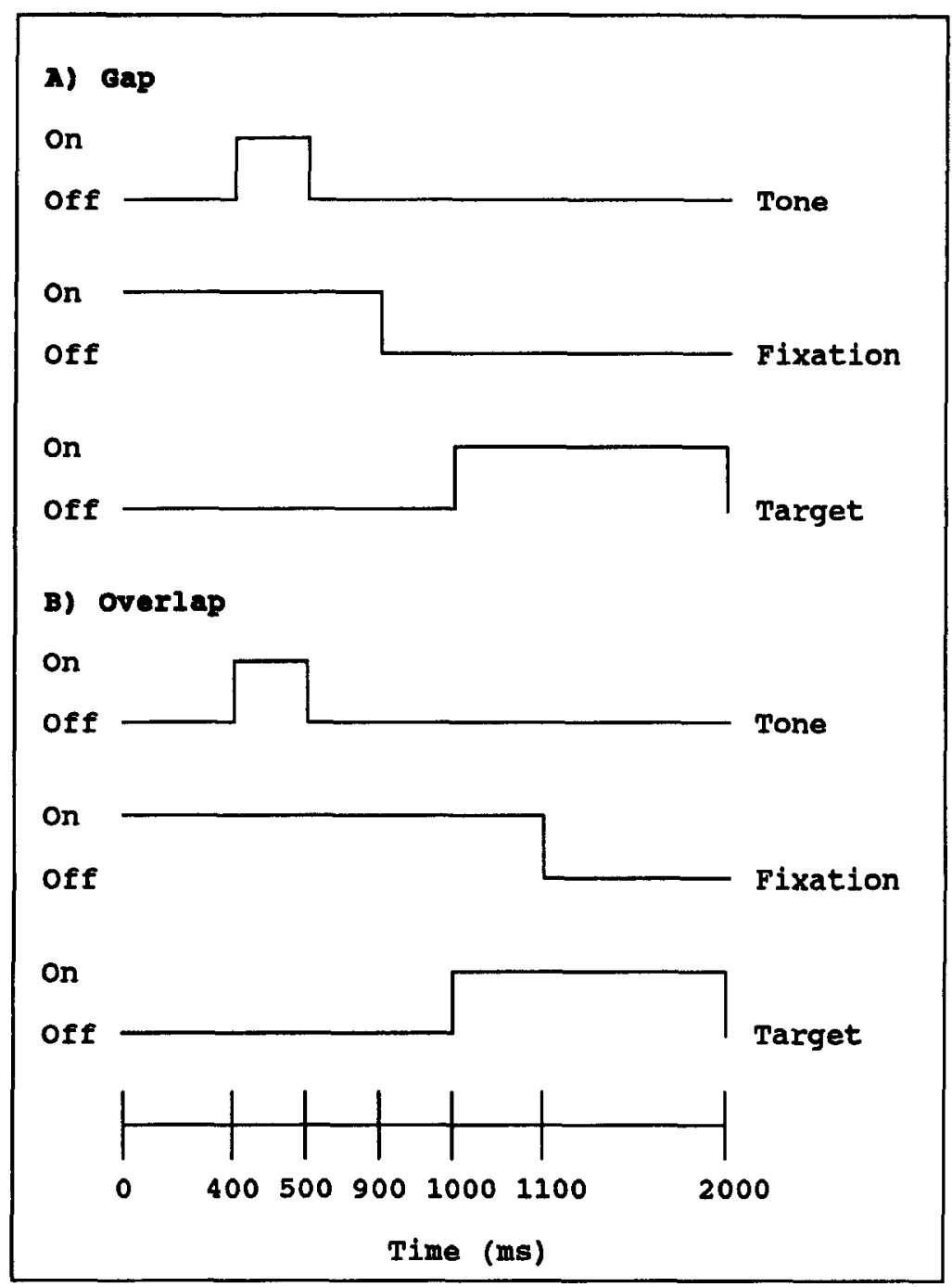

Figure 2. In the gap condition (A), the offset of fixation preceded the target onset (100-msec interstimulus interval [ISI] shown here), whereas in the overlap condition (B), fixation offset followed target onset $(-100-\mathrm{msec}$ ISI shown here). In the present experiments, a 100-msec warning tone was presented $400 \mathrm{msec}$ after the beginning of the trial. The offset of the tone occurred $500 \mathrm{msec}$ before the onset of the target.

type (line vs. numeric). Display type and search type were blocked within a session. The participants executed prosaccades in all the conditions. The dependent measure was saccadic latency. All the saccades were prosaccades.

In the unilateral search condition, a stimulus array was presented on one side of fixation, and the participants were required to search the array for the target item, while maintaining central fixation. The participants executed a saccade to the center of the array only if a target was detected in the array. The target consisted of a vertical line among distractors that were horizontal lines (easy search), or of the digit 2 among distractors that were $5 \mathrm{~s}$ (difficult search). In the bilateral search condition, a stimulus array was presented on both sides of fixation. One side of the display consisted of distractors only, whereas the other side consisted of distractors and a target item. The participants were required to search for the target item and execute a saccade to the center of the array containing the target item. ${ }^{1}$ On catch trials, the stimulus array(s) contained only distractors (horizontal lines or $5 \mathrm{~s}$ ), and the participants were instructed to maintain central fixation.

\section{RESULTS AND DISCUSSION}

\section{Experiment 1: Detection Tasks}

The results of Experiment 1 are shown in Figure 3. Mean saccadic latency was shorter in the prosaccade $(196 \mathrm{msec})$ than in the antisaccade $(243 \mathrm{msec})$ conditions. The gap effect was estimated by comparing the mean latency obtained at an ISI of $-500 \mathrm{msec}$ with that obtained at an ISI of $200 \mathrm{msec}$. In the prosaccade condition, a gap effect was evident ( $47 \mathrm{msec})$. This value is comparable 
with that obtained in other studies of the gap effect in which a warning tone was used (see the summary in Forbes \& Klein, 1996). ${ }^{2}$

The size of the gap effect was smaller in the antisaccade conditions $(28 \mathrm{msec})$. The results of the antisaccade condition agree with the findings of Forbes and Klein (1996; cf. Reuter-Lorenz et al., 1991; Reuter-Lorenz et al., 1995).

The results were analyzed statistically, using a 2 (saccade direction) $\times 8$ (ISI) $\times 2$ (stimulus type) repeated measures analysis of variance (ANOVA). The main effect of saccade direction was significant $[F(1,7)=24.02$, $\left.M S_{\mathrm{e}}=5,958.98, p<.002\right]$. The main effect of ISI was significant $\left[F(7,49)=23.79, M S_{\mathrm{e}}=349.94, p<.001\right]$. The only significant interaction was saccade direction $X$ ISI $\left[F(7,49)=3.98, M S_{\mathrm{e}}=226.82, p<.002\right]$. For prosaccade conditions, post hoc analyses (Tukey) of mean saccadic latencies indicated that the latencies for the -500 -msec and -100 -msec (overlap) ISIs were significantly larger than the latencies for all of the other ISIs $(p<.05)$. For antisaccade conditions, post hoc analyses (Tukey) of mean saccadic latencies indicated that the latencies for the -500 -msec overlap ISIs were significantly longer than the latencies for all of the gap ISIs and that the latencies for the -100 -msec overlap ISIs were longer than the latencies for all but the 0 - and 500 -msec gap ISIs $(p<.05)$.

The percentage of rejected trials was $2.1 \%$ in prosaccade and $2.3 \%$ in antisaccade conditions. Typically, no more than two or three trials were rejected per session, mostly owing to minor errors in maintaining fixation.

\section{Experiment 2: Search Tasks}

The results for Experiment 2 are shown in Figure 4. The pattern of results was similar in the unilateral and bilateral conditions. Mean saccadic latency was longer when the participants searched for the digit (difficult search) than when they searched for the line (easy search).

A gap effect was evident in all four curves, ranging in magnitude from 40 to $55 \mathrm{msec}$. These values are similar

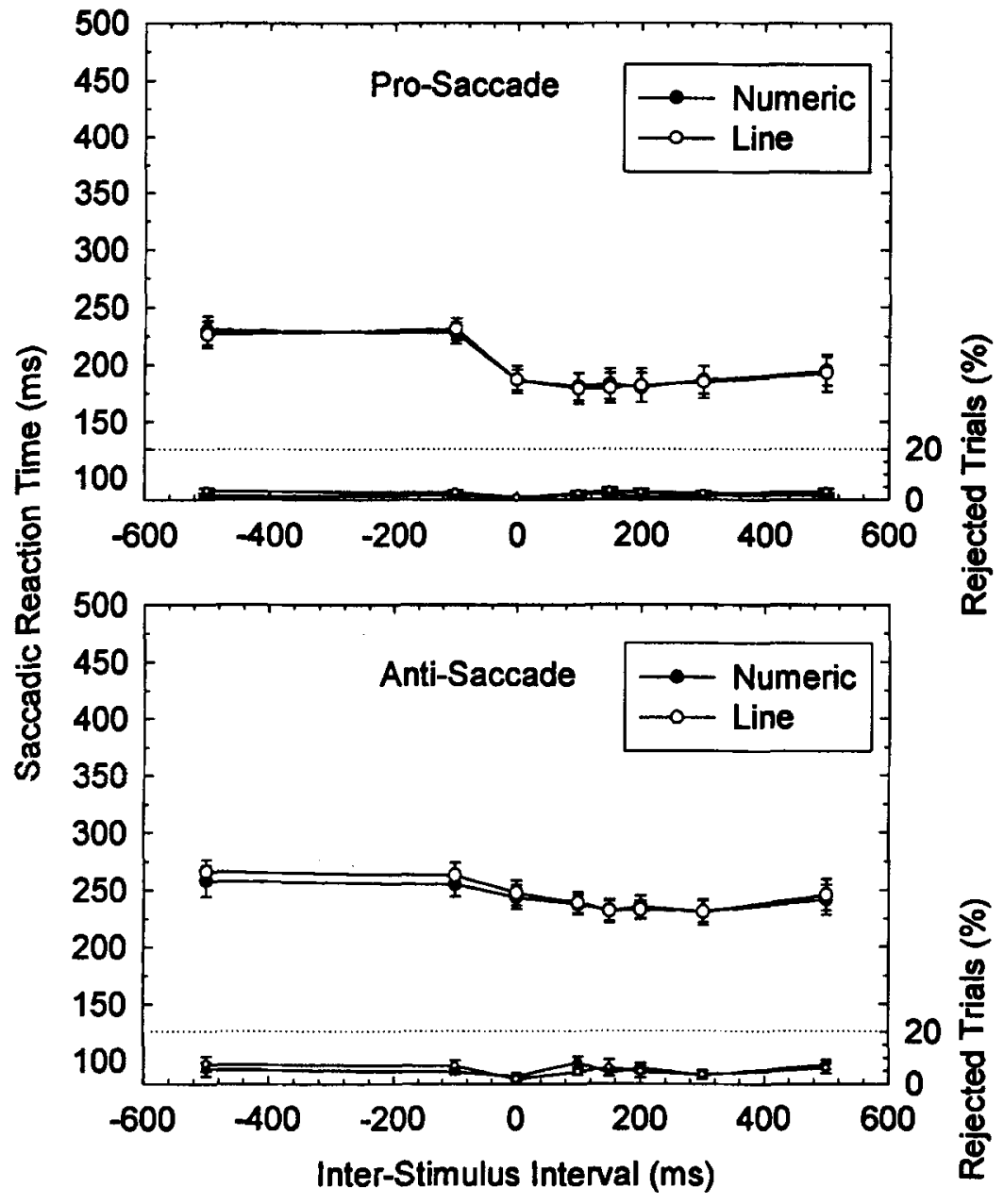

Figure 3. Mean saccadic latencies and percentage of rejected trials for the detection tasks in Experiment 1 are shown in separate panels for prosaccade and antisaccade conditions. Standard errors for each mean are shown. 


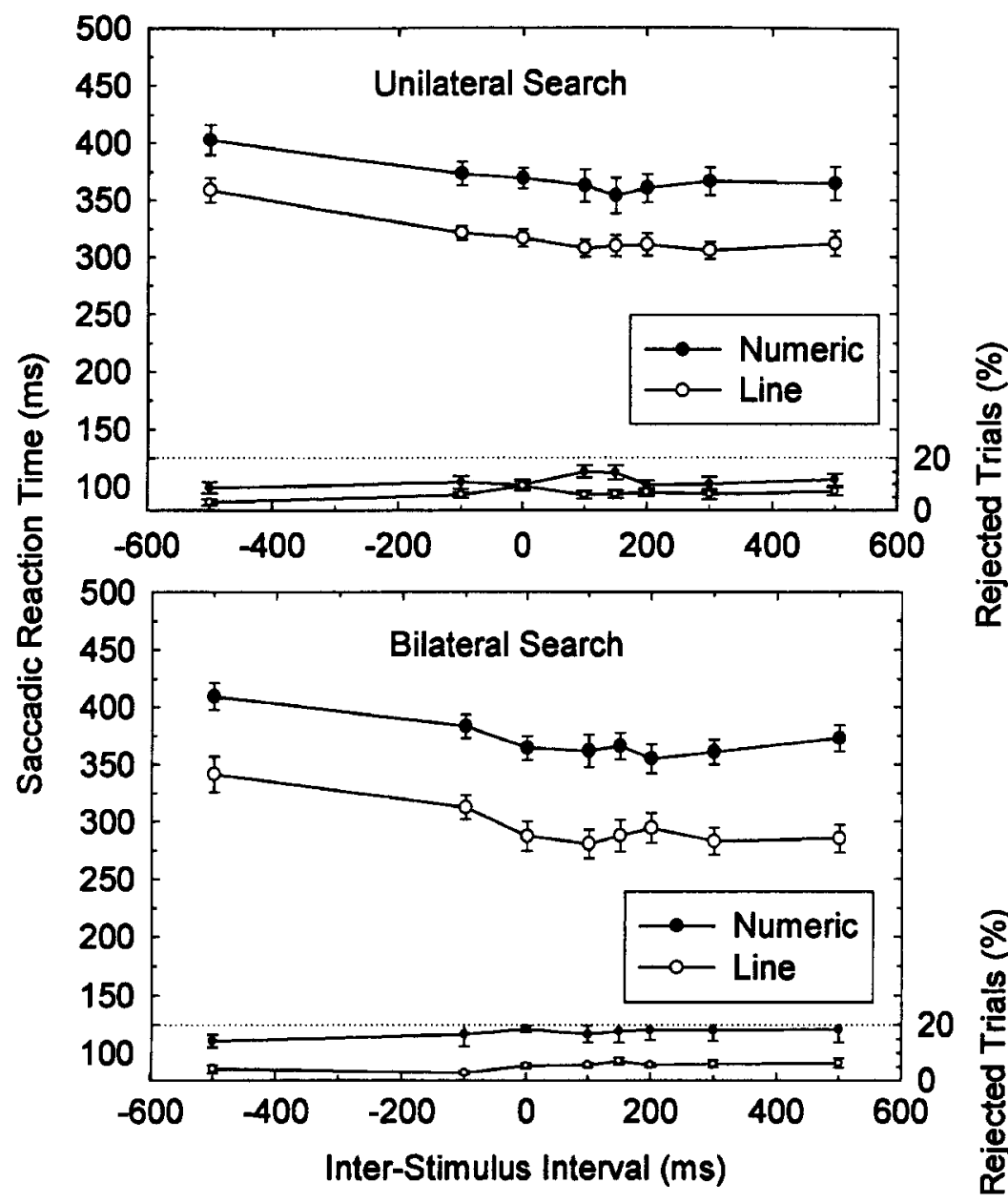

Figure 4. Mean saccadic latencies and percentage of rejected trials for the search tasks in Experiment 2 are shown in separate panels for the unilateral and bilateral search conditions. Standard errors for each mean are shown.

to those obtained in Experiment 1 in the prosaccade condition. As is known, the size of the gap effect can remain roughly constant across changes in the absolute value of mean saccadic latency (Kingstone \& Klein, 1993b). The participants required about $340 \mathrm{msec}$ to initiate a saccade in the present experiment, compared with about $225 \mathrm{msec}$ in Experiment 1 . This was reflected in a gap effect that was evident at ISI $=-100 \mathrm{msec}$, as compared with ISI $=0 \mathrm{msec}$ in Experiment 1, a result that is consistent with our previous findings (Craig, Stelmach, \& Tam, 1994). Given the longer latencies in Experiment 2, the -100 -msec ISI effectively became a gap condition, because the fixation marker was removed from the screen well before the initiation of the saccade. This also explains the apparent difference in the slope between the saccadic reaction time curves in the prosaccade conditions in the two experiments.

The results of Experiment 2 were analyzed, using a 2 (display type) $\times 8$ (ISI) $\times 2$ (search type) repeated mea- sures ANOVA. The main effect of display type (unilateral vs. bilateral) was not significant. The main effect of ISI was significant $\left[F(7,49)=14.05, M S_{\mathrm{e}}=64.60, p<\right.$ $.0001]$. The main effect of search type was significant $\left[F(1,7)=74.98, M S_{\mathrm{e}}=3,398.81, p<.001\right]$. The only interaction that was significant was the two-way interaction between display type and search type $[F(1,7)=$ $\left.8.20, M S_{\mathrm{e}}=1135.47, p<.02\right]$. The interaction occurred because mean saccadic latency in the line search condition was longer in the unilateral than in the bilateral search condition ( 318 and $296 \mathrm{msec}$, respectively). For the serial search condition, the values were much closer $(269$ and $372 \mathrm{msec}$, respectively). A post hoc analysis (Tukey) of the mean saccadic latencies in the unilateral conditions indicated that saccadic latencies for the -500 -msec ISI condition were longer than the latencies for all the other ISIs, for both unilateral and bilateral presentations. As well, there were significant differences between the -100 msec ISI and the 100- and 300-msec ISIs $(p<.05)$, for the 
bilateral presentations. The percentage of rejected trials was $8 \%$ in unilateral and $11 \%$ in bilateral presentations.

\section{GENERAL DISCUSSION}

In the present research, the prediction of the models of Fischer (1987) and of Forbes and Klein (1996), that a gap effect would be observed with both reflexive and voluntary saccades, was examined. This prediction was confirmed in the present research; a gap effect was observed across a range of tasks that varied in the amount of processing required before the execution of a saccade. Furthermore, the magnitude of the gap effect in the search tasks was largely unaffected by the level of difficulty involved in locating the target stimulus. Indeed, the magnitude of the gap effect was roughly constant in all prosaccade conditions, regardless of the absolute value of saccadic latency. The results are consistent with the idea that reflexive and voluntary saccades share a common fixation disengagement stage that is essentially unaffected by higher level processing.

According to the models cited above, fixation disengagement was hypothesized to occur within the rostral superior colliculus, requiring little or no cortical processing (Fischer, 1987; Forbes \& Klein, 1996). On overlap trials, once the target has been located, activity in the fixation cells in the rostral superior colliculus would decrease, whereas activity in the saccade cells in the caudal superior colliculus would increase. At some point at which the activity in the fixation cells was negligible (i.e., disengagement from fixation had occurred) and the activity in the saccade cells was sufficient, a saccade would be executed (Forbes \& Klein, 1996; Munoz \& Wurtz, 1993a, 1993b). On gap trials, the saccade would be facilitated, because removal of the fixation stimulus would lead to a reduction in the activity of fixation cells in the rostral superior colliculus, reaching a minimum about $100-200 \mathrm{msec}$ after the offset of the fixation stimulus (Dorris \& Munoz, 1995). Thus, saccadic latencies would be shorter on gap trials than on overlap trials, because there would be less competition from fixation cells in the rostral superior colliculus. In conclusion, the results of the present study provide strong support for the idea that the fixation stimulus plays an important role in determining saccadic latency.

It is interesting that the participants could not use the onset of the target array to initiate disengagement. After all, the onset of the array provided a signal to the participant that it was time to start searching for the target stimulus and that a saccade was imminent. Since saccadic latencies were substantially longer than $200 \mathrm{msec}$ with the search tasks, there would be sufficient time to complete the disengagement process. This would lead one to predict no effect of fixation offset and a flat saccadic latency curve as a function of ISI. The present results suggest that fixation disengagement occurred only after the target was located. In gap conditions, the oculomotor system would be ready to respond immediately after the tar- get item was located. In overlap conditions, once the target was located, the oculomotor system would respond only after an additional delay of $30-40 \mathrm{msec}$, needed to silence the fixation cells (Munoz \& Wurtz, 1993a).

For completeness, it is useful to consider the role of attention in the gap effect. It has been hypothesized that disengagement of attention, and not disengagement of oculomotor mechanisms, yields the gap effect (Braun \& Breitmeyer, 1988; Mayfrank, Mobashery, Kimmig, \& Fischer, 1986). On this view, attention needs to be in a disengaged state (not engaged at fixation or at a target location), in order for there to be reduced saccadic latencies. With regard to this point, in Experiment 2, attention was always engaged either at fixation or at the target array. As such, the attentional disengagement hypothesis would have predicted no effect of fixation offset and a flat saccadic latency curve as a function of ISI, a prediction that is inconsistent with the results of the current research. Although it is intuitively appealing, the attentional disengagement hypothesis has not been supported in the research literature and, thus, cannot be regarded as a viable explanation of the gap effect (Kingstone \& Klein, 1993a; Klein et al., 1995; Tam \& Stelmach, 1993). Our results support the interpretation that the gap effect is a relatively low-level effect in the visual system that is largely independent of higher level cognitive influences.

\section{REFERENCES}

Abrams, R. A., OONK, H. M., \& Pratt, J. (1998). Fixation point offsets facilitate endogenous saccades. Perception \& Psychophysics, 60, 201-208.

Braun, D., \& Breitmeyer, B. G. (1988). Relationship between directed visual attention and saccadic reaction times. Experimental Brain Research, 73, 546-552.

Craig, G. L., Stelmach, L. B., \& TAM, W. J. (1994, June). The gap effect: Examining delayed saccadic responses. Poster presented at the meeting for the Canadian Society for Brain, Behaviour and Cognitive Psychology, Vancouver, BC.

DorRIs, M. C., \& Munoz, D. P. (1995). A neural correlate for the gap effect on saccadic reaction times in monkey. Journal of Neurophysiology, 73, 2558-2562.

FINLEY, G. (1985). A high-speed point plotter for vision research. Vision Research, 25, 1993-1997.

FISCHER, B. (1987). The preparation of visually guided saccades. Review of Physiology, Biochemistry, \& Pharmacology, 106, 1-35.

FisCHER, B., \& RAMSPERGER, E. (1984). Human express saccades: Extremely short reaction times of goal directed eye movements. Experimental Brain Research, 57, 191-195.

Fischer, B., \& RAMSPERGER, E. (1986). Human express saccades: Effects of randomisation and daily practice. Experimental Brain Research, 64, 569-578.

Fischer, B., \& WEBER, H. (1992). Characteristics of "anti" saccades in man. Experimental Brain Research, 89, 415-424.

FISCHER, B., \& WEBER, H. (1993). Express saccades and visual attention. Behavioral \& Brain Sciences, 16, 553-610.

Forbes, K., \& KLEIN, R. M. (1996). The magnitude of the fixation offset effect with endogenously and exogenously controlled saccades. Journal of Cognitive Neuroscience, $8,344-352$.

Hallett, P. E., \& ADAms, B. D. (1980). The predictability of saccadic latency in a novel voluntary oculomotor task. Vision Research, 20 , 329-339.

IrWIN, D. E., \& CarLson-Radvansky, L. A. (1996). Cognitive suppression during saccadic eye movements. Psychological Science, 7, 83-88. Kingstone, A., \& KLein, R. M. (1993a). Visual offsets facilitate sac- 
cadic latency: Does predisengagement of visuospatial attention mediate this gap effect? Journal of Experimental Psychology: Human Perception \& Performance, 19, 1251-1265.

Kingstone, A., \& Klein, R. M. (1993b). What are human express saccades? Perception \& Psychophysics, 54, 260-273.

KLein, R., TAYlor, T., \& Kingstone, A. (1995). Against a role for attentional disengagement in the gap effect: A friendly amendment to Tam and Stelmach (1993). Perception \& Psychophysics, 57, 573-577.

Lynch, J. C., Mountcastle, V. B., Talbot, W. H., \& Yin, T. C. T. (1977). Parietal lobe mechanisms for directed visual attention. Journal of Neurophysiology, 40, 362-389.

Mayfrank, L., Mobashery, M., Kimmig, H., \& Fischer, B. (1986). The role of fixation and visual attention in the occurrence of express saccades in man. European Archives of Psychiatry \& Neurological Sciences, 235, 269-275.

MunOz, D., \& WURTZ, R. (1992). Role of the rostral superior colliculus in active visual fixation and execution of express saccades. Journal of Neurophysiology, 67, 1000-1002.

Munoz, D., \& WURTz, R. (1993a). Fixation cells in monkey superior colliculus: I. Characteristics of cell discharge. Journal of Neurophysiology, 70, 559-575.

MUNOZ, D., \& WURTZ, R. (1993b). Fixation cells in monkey superior colliculus: II. Reversible activation and deactivation. Journal of Neurophysiology, 70, 576-589.

REULEN, J. P. H. (1984). Latency of visually evoked saccadic eye movements: I. Saccadic latency and the facilitation model. Biological Cybernetics, 50, 251-263.

Reuter-Lorenz, P. A., Hughes, H. C., \& Fendrich, R. (1991). The reduction of saccadic latency by prior offset of the fixation point: An analysis of the gap effect. Perception \& Psychophysics, 49, 167-175.

Reuter-Lorenz, P. A., OONK, H. M., Barnes, L. L., \& Hughes, H. C. (1995). Effects of warning signals and fixation point offsets on the latencies of pro- versus anti-saccades: Implications for an interpretation of the gap effect. Experimental Brain Research, 103, 287-293.
Ross, L. E., \& Ross, S. M. (1980). Saccadic latency and warning signals: Stimulus onset, offset, and change as warning events. Perception \& Psychophysics, 27, 251-257.

SASLOW, M. G. (1967). Effects of components of displacement-step stimuli upon latency for saccadic eye movements. Journal of the $O p$ tical Society of America, 57, 1024-1029.

TAM, W. J., \& ONO, H. (1994). Fixation disengagement and eye-movement latency. Perception \& Psychophysics, 56, 251-260.

TAM, W. J., \& STELMACH, L. B. (1993). Viewing behaviour: Ocular or attentional disengagement. Perception \& Psychophysics, 54, 211-222.

Weban-Smith, M. G., \& Findlay, J. M. (1991). Express saccades: Is there a separate population in humans? Experimental Brain Research, 87, 218-222.

Weber, H., Aiple, F., Fischer, B., \& Latanov, A. (1992). Dead zone for express saccades. Experimental Brain Research, 89, 214-222.

WEBER, H., BISCALDI, M., \& FISCHER, B. (1995). Intertrial effects of randomization on saccadic reaction times in human observers. Vision Research, 35, 2615-2642.

\section{NOTES}

1. Mean saccadic amplitude among all the prosaccade conditions in the present paper ranged from $2.0^{\circ}$ to $2.2^{\circ}$, with a standard deviation of $0.3^{\circ}$. Mean saccadic amplitude was slightly greater in the antisaccade condition, at $2.6^{\circ}-2.7^{\circ}$, with a standard deviation of $0.6^{\circ}$.

2. Although not directly pertinent to the goals of the present paper, we examined the shape of the saccadic reaction time distributions in gap conditions. Bimodal distributions, which are diagnostic of express saccades, were evident for 4 of the 8 subjects.

(Manuscript received November 4, 1997; revision accepted for publication June 10, 1998.) 